\title{
Synthesis of the lipoteichoic acid of the Streptococcus species DSM 8747
}

\author{
Yan Qiao ${ }^{a}$, Buko Lindner ${ }^{b}$, Ulrich Zähringer ${ }^{b}$, Peter Truog ${ }^{c}$, Richard R. Schmidt ${ }^{a, *}$ \\ ${ }^{a}$ Fachbereich Chemie, Universität Konstanz, Fach 725. D-78457-Konstanz, Germany \\ ${ }^{\mathrm{b}}$ Laborgruppe Immunchemie, Forschungszentrum Borstel, Leibniz-Zentrum für Medizin und Biowissenschaften, Parkallee 1-40, D-23845-Borstel, Germany \\ 'LUNAMed AG, CH-7000 Chur, Switzerland
}

\section{Keywords:}

Lipoteichoic acid (LTA)

Synthesis

Glycosidation

Glycophospholipids

Gram-positive bacteria

\begin{abstract}
A B S T R A C T
The lipoteichoic acid (LTA) of the Streptococcus species DSM 8747 consists of a $\beta$-D-galactofuranosyl diacylglycerol moiety (with different acyl groups) that is linked via 6-0 to a poly(glycerophosphate) backbone; about $30 \%$ of the glycerophosphate moieties carry at 2-O hydrolytically labile D-alanyl residues. As typical LTA for this array of compounds LTA 1a was synthesized. To this end, from D-galactose the required galactofuranosyl building block 5 was obtained. The anomeric stereocontrol in the glycosylation step with 1,2-0-cyclohexylidene-sn-glycerol (4) was based on anchimeric assistance, thus finally leading to the unprotected core glycolipid 16. Regioselective protection and deprotection procedures permitted the defined attachment of the pentameric glycerophosphate 3 to the 6-hydroxy group of the galactose residue. Introduction of four D-alanyl residues led after global deprotection and purification to target molecule 1a possessing on average about two $D$-alanyl residues at 2-O of the pentameric glycerophosphate backbone, thus being in close accordance with the structure of the natural material. (c) 2010 Published by Elsevier Ltd.
\end{abstract}

\section{Introduction}

A unique lipoteichoic acid (LTA) was isolated from the cell wall of the Streptococcus species DSM 8747 and structurally assigned by Roethlisberger et al. ${ }^{12}$ (Fig. 1,1 ). The LTA comprises a linear (1-3)-linked poly(glycerophosphate) chain which is partly substituted with 2-O-D-alanyl residues; this part is phosphodiester linked to the 6-hydroxy group of 3-0-( $\beta$-D-galactofuranosyl)-1,2di-O-acyl-sn-glycerol; thus, 1 belongs to the type I LTA structures. ${ }^{3}$ Noteworthy for the structure of $\mathbf{1}$ is the average length of only about ten glycerophosphate residues with about three $\mathrm{D}$-alanyl residues attached and the core of the lipid anchor is proposed to possess the rare mono-hexosyl-1,2-diacyl-sn-glycerol structure. This Gram-positive bacterium is genetically closely related to Streptococcus pneumoniae that encomprises in its cell wall a structurally totally different LTA, belonging to type IV LTA. ${ }^{3 \mathrm{~b}}$ A total synthesis of this LTA has been recently accomplished, ${ }^{4}$ thus the previous structural assignment was confirmed, yet the biological studies with this synthetic material led to unexpected results. 4.5 Hence, the synthesis of the Streptococcus species DSM 8747 was of great interest as well.

As indicated in Figure 1, LTA 1 varies in the acyl residues in the chain length of the glycerophosphate backbone, as well as in the number and location of the attached D-alanyl residues that are hydrolytically labile and cleaved rapidly even at $\mathrm{pH} 8.5 .6$ Our

\footnotetext{
* Corresponding author. Tel.: +497531882538 ; fax: +497531883135. E-mail address: richard.schınidt@uni-konstanz.de (R.R. Schmidt).
}

previous studies of Staphylococcus aureus revealed that a backbone length of about five to six residues is sufficient for biological activity. ${ }^{7}$ Hence, we decided to synthesize LTA 1a having five glycerophosphate moieties with up to four D-alanyl residues attached before final deprotection. Thus, the hydrolytic lability of the D-alanyl residues and their importance for biological activity has been taken into account and after workup of the target compound at least the average number of $\mathrm{D}$-alanyl residues should be available ( $1 \mathrm{a} \approx 0.3 n=1.5 \mathrm{D} m$ Ala residues). For the acyl chain, myristoyl $\left(C_{14}\right)$ residues were chosen.

\section{Results and discussion}

The synthesis design of LTA 1a is outlined in the retro-synthetic scheme (Scheme 1). For a convergent synthesis of the molecule, a disconnection between the glycolipid core and the oligo(glycerophosphate) moiety is proposed leading to intermediates $\mathbf{2}$ and 3 ; their further disintegration furnishes building blocks 4-7. Thus, for the $\beta$-linkage in intermediate 2 neighbouring group participation for the galactofuranosyl donor $\mathbf{5}$ is employed. For convenience, the totally benzoylated donor is used in the reaction with known 1,2 -cyclohexylidene-sn-glycerol $(4)^{8}$ as acceptor, thus requiring in the following steps for the galactosyl residue an exchange of protecting groups, that is, temporary protection at 6-O and benzyl protection at 2-, 3-, and 5-0. Known intermediate $3^{7,9}$ with temporary 2-O-MPM (4-methoxyphenylmethyl) protection permits selective cleavage in the presence of $O$-benzyl protecting groups, thus allowing introduction of the D-alanyl residues as the second 


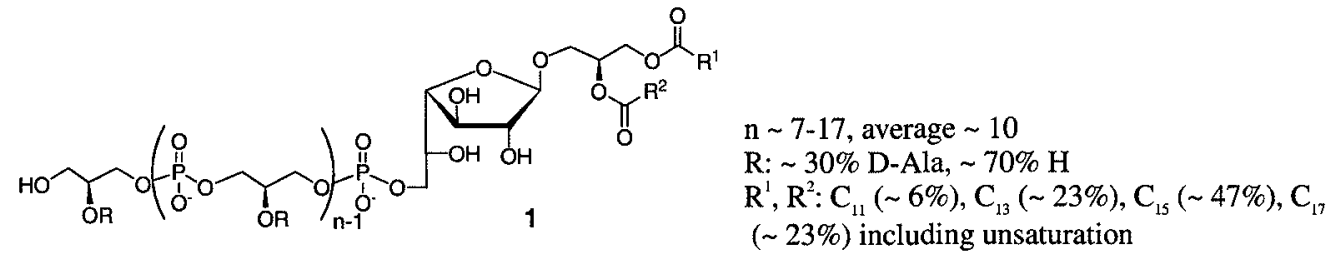

Figure 1. Structure of LTA found in Streptococcus species DSM 8747.

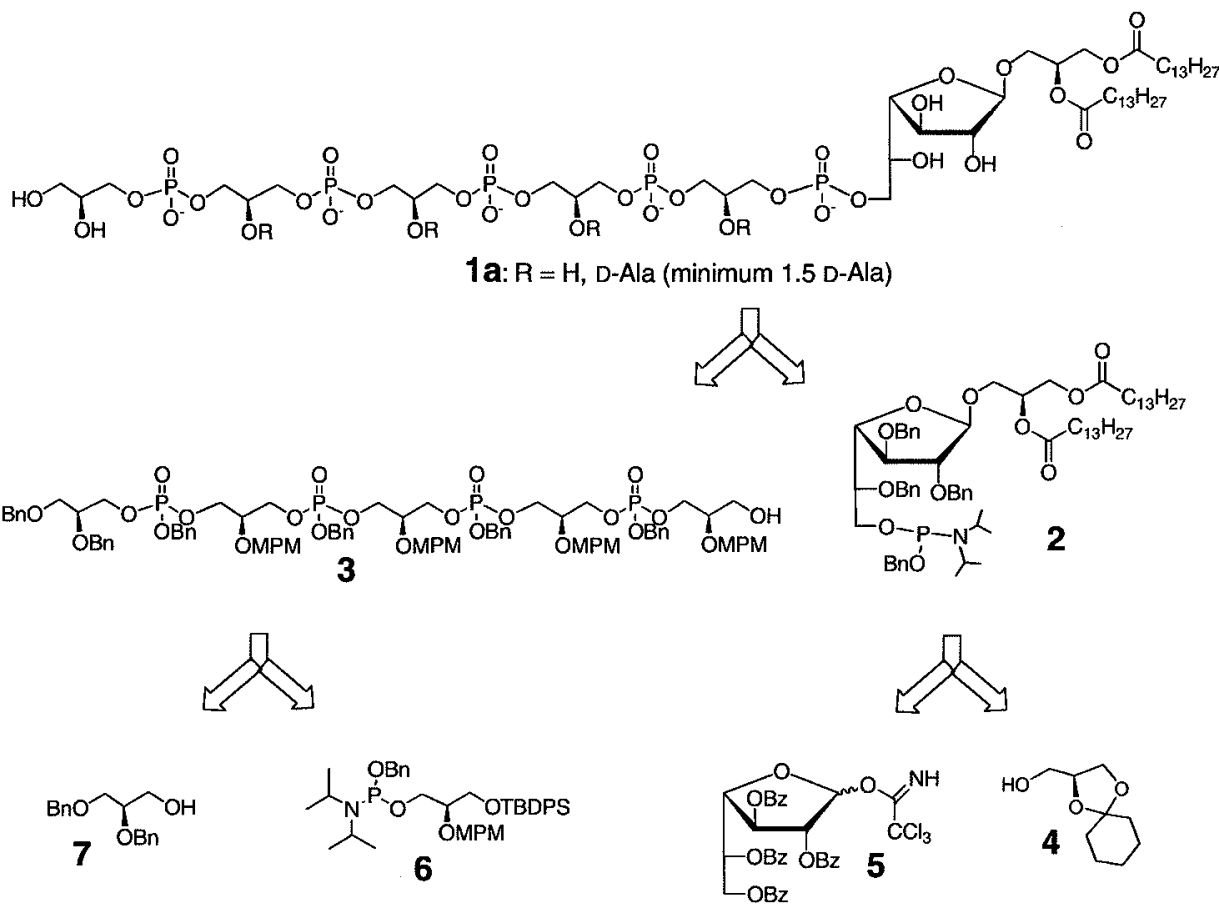

Scheme 1. Retro-synthetic scheme for the synthesis of target molecule 1a.

last step and thereafter global hydrogenolytic O-debenzylation under mild conditions. This way, as previously found by us in the synthesis of S. aureus LTA, ${ }^{7}$ another type I LTA, no or little loss of the hydrolytically labile D-alanyl residues is expected. Therefore, the previously prepared building blocks $6^{7,9,10}$ and $7^{7,8,11}$ will be employed for the synthesis of 3.

For the synthesis of the phosphitylated glycolipid core structure 2 , D-galactose was transformed into octyl $\beta$-D-galactofuranoside (8) and its per- 0 -benzoyl derivative $\mathbf{9}$ following a reported procedure (Scheme 2). ${ }^{12}$ Acetolysis of $\mathbf{9}$ with acetic anhydride/sulfuric acid furnished, contrary to the literature reports claiming the requirement of trifluoroacetic anhydride for the acidolysis, ${ }^{13}$ the desired 1-O-acetyl protected intermediate $10^{14}$ that was transformed by treatment with $\mathrm{HBr}$ and acetic anhydride and then with water into 1-O-unprotected intermediate 11. ${ }^{15}$ Reaction with trichloroacetonitrile in the presence of DBU as base afforded quantitatively O-galactofuranosyl trichloroacetimidate $5^{16}$ as anomeric mixture. Glycosylation of $\mathbf{4}^{8}$ with TMSOTf as catalyst in dichloromethane as solvent afforded the desired $\beta$-D-galactofuranoside $\mathbf{1 2}$ in high yield. The NMR data of $12\left[{ }^{1} \mathrm{H}\right.$ NMR: $\delta_{\mathrm{H}-1 \mathrm{a}}=5.29(\mathrm{~s}) ;{ }^{13} \mathrm{C}$ NMR: $\left.\delta_{\mathrm{C}-1}=105.96, \quad J_{\mathrm{CH}}=176.7 \mathrm{~Hz}\right]$ are in accordance with those reported for $\beta$-D-galactofuranosides. ${ }^{15,17}$ In order to avoid rearrangement reaction in this molecule, the benzoyl groups were removed under Zemplén conditions and then replaced by benzyl groups to give intermediate 13. Cleavage of the cyclohexylidene group in the presence of ethylene glycol as nucleophile and p-toluenesulfonic acid ( $p$-TsOH) as catalyst afforded glycerol derivative 14. Treatment of this compound with myristic acid in the presence of dicyclohexylcarbodiimide (DCC) as condensing agent and 4-dimethylaminopyridine (DMAP) as catalyst provided diacylglycerol derivative 15. Hydrogenlytic O-debenzylation of 15 with $\mathrm{Pd} / \mathrm{C}$ as catalyst in the presence of trifluoroacetic acid (TFA) gave the glycolipid 16 that was of interest for comparisons in the biological studies.

For the regioselective attachment of the glycerophosphate backbone to the glycolipid anchor 16, 6-0-silylation with tertbutyldimethylsilyl chloride (TBDMSCl) with $\mathrm{NEt}_{3}$ as base and DMAP as catalyst was carried out affording selectively protected compound 17 (Scheme 3). Following O-benzylation with benzyl bromide and $\mathrm{NaH}$ as base gave fully protected derivative 18 that was desilylated by treatment with tetra- $n$-butylammonium fluoride (TBAF), thus affording 6a-O-unprotected intermediate 19. Reaction with bis(diisopropylamino) benzyloxyphosphine ${ }^{18}$ in the presence of bis(diisopropyl) ammonium tetrazolide as catalyst gave the desired 6-O-phosphitylated $\beta$-D-galactofuranosyl-diacylglycerol 2.

Reaction of previously synthesized $\mathbf{3}^{7,9}$ with $\mathbf{2}$ in the presence of tetrazole as catalyst afforded the phosphite triester intermediate that was oxidized with tert-butyl hydroperoxide to the corresponding phosphate $\mathbf{2 0}$ and obtained as mixture of diastereomers. 

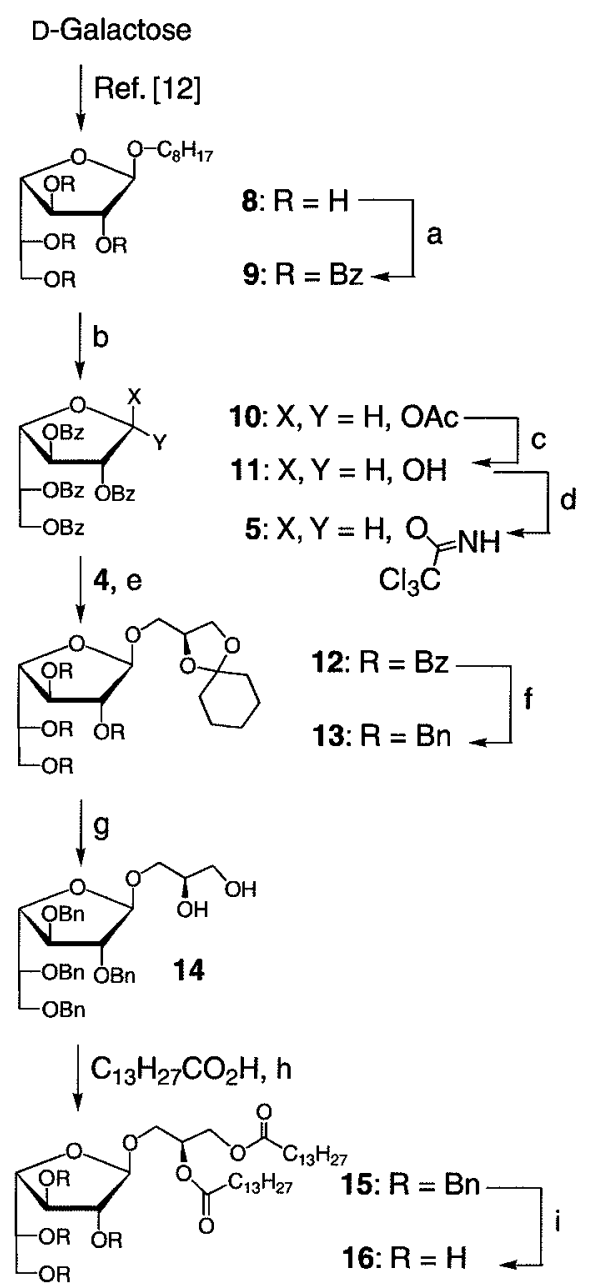

Scheme 2. Synthesis of the core glycolipid 16. Reagents and conditions: (a) $\mathrm{BzCl}$, Py (qu); (b) $\mathrm{Ac}_{2} \mathrm{O}, \mathrm{H}_{2} \mathrm{SO}_{4}, \mathrm{O}^{\circ} \mathrm{C}$ (95\%); (c) $\mathrm{HBr}, \mathrm{Ac}_{2} \mathrm{O}, \mathrm{DCM}, \mathrm{H}_{2} \mathrm{O}$ (90\%); (d) $\mathrm{CCl}_{3} \mathrm{CN}, \mathrm{DBU}$ DCM (qu); (e) TMSOTf (0.1 equiv), DCM (89\%); (f) $\mathrm{NaOMe,} \mathrm{MeOH;} \mathrm{then} \mathrm{BnBr}, \mathrm{NaH}$, DMF (96\%); (g) p-TsOH, DCM-CH ${ }_{3} \mathrm{CN}$, ethylene glycol (76\%); (h) DCM, DMAP, DCC (qu); (i) $\mathrm{Pd} / \mathrm{C}, \mathrm{H}_{2}$, TFA (cat.), EtOAc-MeOH (90\%)

Oxidative cleavage of the MPM groups with ceric ammonium nitrate (CAN) in an acetonitrile/toluene/water mixture liberated four hydroxy groups of the glycerophosphate backbone furnishing compound 21. For the attachment of the D-alanyl residues, 21 was reacted with the triethylammonium salt of $N$-benzyloxycabonyl (Cbz) protected D-alanine and benzotriazol-1-yl-oxytripyrrolidinophosphonium hexafluorophosphate (PyBOP) as coupling reagent in the presence of $\mathrm{N}$-methylimidazole, thus affording compound $\mathbf{2 2}$ as fully protected derivative of the target molecule. Hydrogenolytic cleavage of the six $O$-benzyl and four $Z$ groups was performed with Pearlman's catalyst in a mixture of dichloromethane/methanol/ water, thus providing after hydrophobic interaction chromatography (HIC) with an ammonium acetate/n-propanol gradient (from 85:15 to 40:60) pure target compound 1a with about two D-alanyl residues attached. This material will be used for biological studies.

The charge deconvoluted ESI FT-ICR mass spectrum obtained in the negative ion mode revealed molecular mass peaks being in agreement for $1 \mathrm{a}$ with zero to four alanyl residues with the highest intensity for two alanyl residues. Hence, as expected, alanyl residues have been lost in the deprotection and/or purification procedure. The MS data confirm that on average at least two alanyl residues are attached.
All attempts to record well resolved ${ }^{1} \mathrm{H}$ NMR spectra in deuterated THF, DMSO, water, methanol and mixtures of these solvents remained unsuccessful due to micelle and aggregate formation of the glycolipid part. The solvent for HIC (propanol/water, 1:1) was found to be the best for the NMR experiments at concentrations that were sufficient for heteronuclear correlated ${ }^{1} \mathrm{H},{ }^{13} \mathrm{C},{ }^{31} \mathrm{P}$ NMR spectroscopy. This way, a peak assignment by homo- and heteronuclear correlated NMR spectroscopy was possible (Table 1). The NMR data are in good accordance with those reported for the natural material, ${ }^{1,2}$ thus confirming the previous structure assignment. In the ${ }^{1} \mathrm{H}$ MNR spectra of 1a three different signals for $2-\mathrm{H}$ of the glycerol (Gro) residue were obtained. One signal at $\delta 5.38$ $\left(\mathrm{C}-2^{\mathrm{Gro}}=74.9\right)$ is assigned to $\mathrm{O}$-acylated $2-\mathrm{H}$ of the diacylglycerol (DAG) residue. The signal at $\delta 5.30\left(\mathrm{C}-2^{\mathrm{Gro}}=70.1\right)$ originates from the glycerophosphate repeating units having 2-O-alanyl residues and the signal at $\delta 4.33\left(\mathrm{C}-2^{\mathrm{Gro}}=75.0\right)$ is due to O-unsubstituted glycerophosphate residues. In agreement with this also for $2-\mathrm{H}$ of the alanyl residues two different signals were observed: One signal at $\delta 4.26\left(\mathrm{C}-2^{\mathrm{Ala}}=49.5\right)$ is due to ester bound 2-0-alanyl residues and the other signal at $\delta 3.73\left(\mathrm{C}-2^{\mathrm{Ala}}=51.2\right)$ originates from nonbound alanine, that is known to be co-eluting with LTA form the HIC column. ${ }^{6}$ From the signals at $\delta 5.38$ and 5.30 an intensity ratio of about 1:2 was observed, indicating that 1a contains about two $O$-alanyl residues. This is in agreement with the ESI-MS data. Hence, about two 0 -alanyl residues were lost in the deprotection and/or purification procedure of $1 \mathrm{a}$ that seems to be more sensitive to O-de-alanylation than the LTA of S. aureus. ${ }^{7,9}$

\section{Summary}

In conclusion, following the retro-synthetic design the LTA 1a of the Streptococcus species DSM 8747 was successfully synthesized. The expected partial hydrolytic cleavage of D-alanyl residues during final deprotection and/or purification could be compensated by introducing beforehand a higher degree of alanylation. Thus, the ratio of the constituents of 1 a with on average two D-alanyl residues is in good agreement with the natural compound.

\section{Experimental details}

\subsection{General}

Solvents were dried according to standard procedures. NMR spectroscopic measurements were performed at $22^{\circ} \mathrm{C}$ with Bruker DRX 600. Bruker Avance 600 cryo, Bruker 400 Avance, Varian Mercury 300 and Bruker AC250 spectrometers. Tetramethylsilane (TMS) or the resonances of the deuterated solvents were used as internal standards: $\mathrm{CDCl}_{3},(\delta=7.24 \mathrm{ppm})$ was used as an external standard, $85 \%$ of phosphoric acid was used as an external standard for ${ }^{31} \mathrm{P}$ spectra. MALDI mass spectra were recorded with a Kratos Kompact MALDI II spectrometer; 2,5-dihydroxybenzoic acid (DHB) or $p$-nitroaniline and $\mathrm{NaI}$ were used as matrices for positive mode measurements, and trihydroxyacetophenone (THAP) was used as the matrix for negative mode measurements. HRMS spectra were recorded with a Bruker ESI-MS mass spectrometer. High resolution Electrospray lonization Fourier Transform Ion Cyclotron Mass Spectrometry (ESI FT-ICR MS) for 1a was performed in the negative ion mode on a 7 Tesla APEX Qe (Bruker Daltonics). Samples preparation and instrumental settings were the same as described previously. ${ }^{4}$ Optical rotations were measured at $22^{\circ} \mathrm{C}$ with a Büchi Polar-Monitor using the sodium D line. Thin-layer chromatography (TLC) was performed on Merck Silica Gel (60 $\mathrm{F}_{254}$ ) plastic plates; compounds were visualized by treatment with a solution of $\left(\mathrm{NH}_{4}\right)_{6} \mathrm{Mo}_{7} \mathrm{O}_{24} \cdot 4 \mathrm{H}_{2} \mathrm{O}(2 \mathrm{~g} \mathrm{~g})$ and $\mathrm{Ce}\left(\mathrm{SO}_{4}\right)_{2}(0.4 \mathrm{~g})$ in sulfuric acid $(10 \%, 400 \mathrm{~mL})$ and then by heating to $120^{\circ} \mathrm{C}$. Flash 

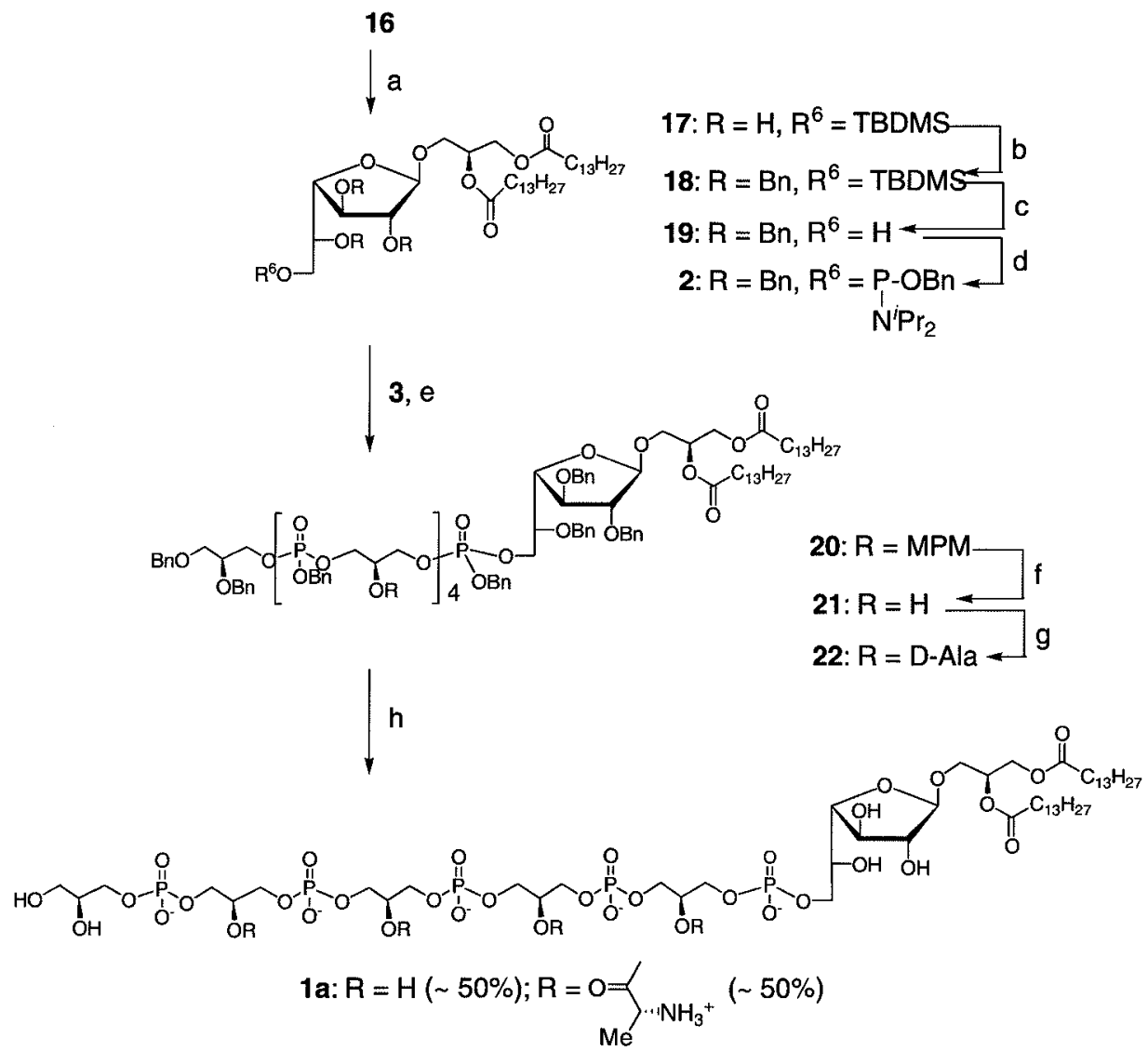

Scheme 3. Synthesis of the target molecule 1a. Reagents and conditions: (a) TBDMSCl, DMAP, Et ${ }_{3} \mathrm{~N}, \mathrm{DCM}(63 \%)$; (b) BnBr, NaH, DMF (90\%); (c) TBAF, DCM (76\%); (d)

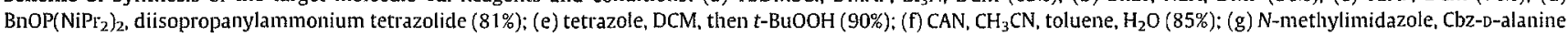
triethylammonium salt, PyBOP, DCM (74\%); (h) $\mathrm{Pd}(\mathrm{OH})_{2}, \mathrm{H}_{2}$. DCM/MeOH/H $/ \mathrm{H}_{2} \mathrm{O}(7: 3: 2)(28 \%)$.

chromatography was performed on MN Silica Gel 60 (230-400 mesh) at a pressure of 0.2 bar. Target molecules were purified by hydrophobic interaction chromatography on octyl-sepharose as the stationary phase and the elution phase was used as a gradient of propanol (15-60\%) in $0.1 \mathrm{~mol} / \mathrm{L}$ anmonium acetate buffer ( $\mathrm{pH}$ $4.8)$.

\subsubsection{2,3,5,6-Tetra-0-benzoyl- $\alpha, \beta$-D-galactofuranosyl acetate (10)}

To a solution of $9(2.1 \mathrm{~g}, 3 \mathrm{mmol})$ in $\mathrm{CH}_{2} \mathrm{Cl}_{2}(21 \mathrm{~mL})$ at $0{ }^{\circ} \mathrm{C}$, acetic anhydride $(1.13 \mathrm{~mL})$ and concd $\mathrm{H}_{2} \mathrm{SO}_{4}(0.08 \mathrm{~mL})$ were added and the mixture stirred for $30 \mathrm{~min}$. The temperature was then allowed to rise to $25^{\circ} \mathrm{C}$ and after $4 \mathrm{~h}$ the reaction mixture was quenched by adding excess satd $\mathrm{NaHCO}_{3}$. The mixture was extracted with ethyl acetate and the organic layer was washed successively with ice water, aqueous $\mathrm{NaHCO}_{3}$ and water and then dried over $\mathrm{MgSO}_{4}$ and concentrated in vacuo to give crude 10. After purification as described ${ }^{14}$ the NMR data are identical with those reported in the literature ${ }^{14}$ for $\mathbf{1 0}$.

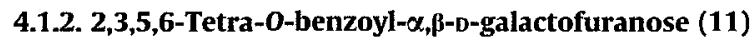

Compound $\mathbf{1 1}$ was obtained from $\mathbf{1 0}$ following the reported procedure. ${ }^{15}$

\subsubsection{0 -(2,3,5,6-Tetra- 0 -benzoyl- $\alpha, \beta$-D-galactofuranosyl) trichloroacetimidate (5)}

Reaction of 11 with $\mathrm{CCl}_{3} \mathrm{CN}$ and $\mathrm{DBU}$ in $\mathrm{CH}_{2} \mathrm{Cl}_{2}$ at room temperature afforded known $\mathbf{5}^{16}$ in practically quantitative yield.
4.1.4. 3-0-(2,3,5,6-Tetra-0-benzoyl- $\alpha, \beta$-D-galactofuranosyl)-1,2$O$-cyclohexylidene-sn-glycerol (12)

To a solution of 5 ( $2.6 \mathrm{~g}, 3.5 \mathrm{mmol})$ and 1,2-0-cyclohexylidenesn-glycerol (4) ( $750 \mathrm{mg}, 4.3 \mathrm{mmol}$; commercial material) in $\mathrm{CH}_{2} \mathrm{Cl}_{2}$ $(60 \mathrm{~mL})$ was added TMSOTf $(70 \mu \mathrm{L}, 0.35 \mathrm{mmol})$ under argon. The reaction was stirred for $3 \mathrm{~h}$ and then quenched with $\mathrm{NEt}_{3}$ $(0.2 \mathrm{~mL})$. The solvent was evaporated in vacuo. Flash silica gel column chromatography (petroleum ether/ethyl acetate $=5: 1$ ) gave $12(2.2 \mathrm{~g}, 89 \%)$ as oil. ${ }^{1} \mathrm{H}$ NMR $\left(400 \mathrm{MHz}, \mathrm{CDCl}_{3}\right): \delta 8.01-7.81(\mathrm{~m}$, $8 \mathrm{H})$. 7.51-7.19 (m, 12H), 6.0-5.97 (m, 1H), $5.55(\mathrm{~d}, 1 \mathrm{H}$, $J=5.0 \mathrm{~Hz}), 5.41(\mathrm{~d}, 1 \mathrm{H}, J=1.0 \mathrm{~Hz}), 5.29(\mathrm{~s}, 1 \mathrm{H}$, anomeric proton $)$, $4.71-4.63(\mathrm{~m}, 2 \mathrm{H}), 4.58(\mathrm{dd}, 1 \mathrm{H}, J=3.8,5.0 \mathrm{~Hz}), 4.25(\mathrm{~m}, 1 \mathrm{H})$, $3.97(\mathrm{dd}, 1 \mathrm{H}, J=6.4,8.3 \mathrm{~Hz}), 3.78-3.71(\mathrm{~m}, 2 \mathrm{H}), 3.52(\mathrm{dd}, 1 \mathrm{H}$, $J=5.7,10.4 \mathrm{~Hz}), 1.54(\mathrm{~m}, 8 \mathrm{H}), 1.33(\mathrm{~m}, 2 \mathrm{H}) .{ }^{13} \mathrm{C} \mathrm{NMR}(101 \mathrm{MHz}$, $\left.\mathrm{CDCl}_{3}\right) \delta 166.12,165.75,165.65,165.40,133.52,133.40,133.26$, $133.12,129.97,129.85,129.73,129.56,129.48,129.03,128.88$, $128.46,128.43,128.41,128.38,110.08,105.96\left(J_{C, H}=176.7 \mathrm{~Hz}\right.$, anomeric carbon), 81.86, 81.63, 77.51, 77.35, 77.23, 77.03, 76.71, $74.02,70.37,67.97,66.27,63.53,36.40,35.01,25.13,24.01$, 23.84. ESI HRMS Calcd for $\mathrm{C}_{43} \mathrm{H}_{42} \mathrm{O}_{12}$ : $[\mathrm{M}+\mathrm{Na}]^{+} \mathrm{m} / \mathrm{z} 773.2568$. Found: $[\mathrm{M}+\mathrm{Na}]^{+} \mathrm{m} / \mathrm{z} 773.2730$.

4.1.5. 3-O-(2,3,5,6-Tetra-o-benzyl- $\beta$-D-galactofuranosyl $)-1,2-0$ cyclohexylidene-sn-glycerol (13)

Compound $12(2.2 \mathrm{~g}, 3 \mathrm{mmol})$ in $\mathrm{MeOH}(20 \mathrm{~mL})$ was treated with $\mathrm{NaOMe}(0.5 \mathrm{~mL}, 1 \mathrm{M}$ in $\mathrm{MeOH})$, then the solvent was removed after $2 \mathrm{~h}$. The crude debenzoylation compound was dissolved in DMF $(20 \mathrm{~mL}), \mathrm{BnBr}(1.8 \mathrm{~mL}, 15 \mathrm{mmol})$ and $\mathrm{NaH}(800 \mathrm{mg}, 60 \%$ in 
Table 1

NMR data of $1 \mathbf{a}^{\mathrm{a}, \mathrm{b}, \mathrm{c}}$

\begin{tabular}{|c|c|c|c|}
\hline \multicolumn{2}{|c|}{$\begin{array}{l}{ }^{1} \mathrm{H} \text { NMR data } \\
\text { Chemical shift }\end{array}$} & \multicolumn{2}{|c|}{$\begin{array}{l}{ }^{13} \mathrm{C} \text { NMR data } \\
\text { Chemical shift }\end{array}$} \\
\hline Proton & $\delta(\mathrm{ppm})$ & Carbon & $\delta(\mathrm{ppm})$ \\
\hline \multicolumn{4}{|l|}{$\beta-D-G a l f$} \\
\hline $1-\mathrm{H}$ & 4.97 & $C-1$ & 108.3 \\
\hline $2-\mathrm{H}$ & 4.04 & $C-2$ & 81.8 \\
\hline $3-\mathrm{H}$ & 4.10 & $c-3$ & 76.8 \\
\hline $4-\mathrm{H}$ & 3.97 & $C-4$ & 84.3 \\
\hline $5-\mathrm{H}$ & 3.89 & $C-5$ & 70.8 \\
\hline $6 a-H$ & $3.9^{c}$ & $C-6$ & 67.0 \\
\hline $6 \mathrm{~b}-\mathrm{H}$ & $3.9^{c}$ & & \\
\hline \multicolumn{4}{|c|}{$\begin{array}{l}\text { Ala (due to heterogeneity, two-different types of Ala and three of Gro were } \\
\text { identified) (see MS) }\end{array}$} \\
\hline & & C-1 & 170.5 \\
\hline $2-\mathrm{H}^{(\text {free })}$ & 3.76 & $\mathrm{C}-2^{\text {(free })}$ & 51.2 \\
\hline $2^{\prime}-\mathrm{H}^{\text {(bound) }}$ & 4.26 & $\mathrm{C}-2^{\prime(\text { bound })}$ & 49.5 \\
\hline $3-\mathrm{H}^{\text {(free) }}$ & 1.59 & $C-3^{\text {(free) }}$ & 16.0 \\
\hline $3^{\prime}-\mathrm{H}^{\text {(bound) }}$ & 1.47 & $C-3^{r(\text { bound })}$ & 16.8 \\
\hline \multicolumn{4}{|l|}{ Gro } \\
\hline $1,1^{\prime}-\mathrm{H}$ & $3.4-3.8^{c}$ & $C-1$ & $64^{c}$ \\
\hline $2-\mathrm{H}^{\mathrm{O}-\mathrm{Acyl}}$ & 5.38 & $C-2^{O-A c y l}$ & 74.9 \\
\hline $2-\mathrm{H}^{\mathrm{O}-\mathrm{Ala}}$ & 5.30 & $C-2^{0 \text {-Ala }}$ & 71.4 \\
\hline $2-\mathrm{H}^{\mathrm{OH}}$ & 4.33 & $\mathrm{C}-2^{\mathrm{OH}}$ & 70.1 \\
\hline $3-H$ & $3.4-3.8^{c}$ & $C-3$ & $65^{c}$ \\
\hline \multicolumn{4}{|c|}{ Fatty acids (14:0) } \\
\hline & & $C-1$ & 170.7 \\
\hline $2-\mathrm{H}$ & 2.28 & $C-2$ & 34.6 \\
\hline $3-\mathrm{H}$ & 1.59 & $C-3$ & 25.3 \\
\hline $4-\mathrm{H}$ & 1.2 & & \\
\hline $4-\mathrm{H}-13-\mathrm{H}$ & $1.02-1.08$ & $C-4 \cdots C-13$ & $22.8 \cdots 24.50^{c}$ \\
\hline $14-\mathrm{H}$ & 0.811 & $C-14$ & 14.3 \\
\hline${ }^{31} \mathrm{p}$ & $+0.82 \mathrm{ppm}(\mathrm{br} \mathrm{s})$ & & \\
\hline
\end{tabular}

${ }^{2}{ }^{1} \mathrm{H},{ }^{13} \mathrm{C},{ }^{31} \mathrm{P}$ NMR $\left[600 \mathrm{MHz} / 150.9 \mathrm{MHz} / 243 \mathrm{MHz}, n\right.$-propanol- $d_{6} / \mathrm{D}_{2} \mathrm{O}$ approx $1: 1(\mathrm{~V} / \mathrm{V}), 300 \mathrm{~K}$; ${ }^{13} \mathrm{C}$ assignments based on HMQC and HSOC-DEPT

${ }^{b}$ Other signals: n-propanol $(\delta, \mathrm{ppm}), \delta_{1-\mathrm{H}} 4.10, \delta_{\mathrm{C}-1} 64.04, \delta_{2-\mathrm{H}} 1.20, \delta_{\mathrm{C}-2} 30.3, \delta_{3-\mathrm{H}}$ $0.78, \delta_{\mathrm{C}-3} 23.5$

Non-resolved signals overlapping with signals of the n-propanol solvent.

oil, $20 \mathrm{mmol}$ ) were added and the reaction mixture stirred overnight, then poured into ice water $(100 \mathrm{~mL})$ and extracted with EtoAc. The obtained extract was washed with water and brine, dried over $\mathrm{MgSO}_{4}$, and evaporated in vacuo. The residue was purified by silica gel column chromatography (petroleum ether/ ethyl acetate $=8: 1)$ to obtain $13(2.5 \mathrm{~g}, 96 \%)$ as oil. ${ }^{1} \mathrm{H}$ NMR $\left(400 \mathrm{MHz}, \mathrm{CDCl}_{3}\right): \delta 7.38-7.29(\mathrm{~m}, 20 \mathrm{H}), 5.12(\mathrm{~s}, 1 \mathrm{H}$, anomeric proton ), 4.77-4.47 (m, 7H), 4.34-4.28 (m, 2H), 4.16-4.14 (m, $1 \mathrm{H})$, 4.07-4.03 (m, 3H), 3.87-3.71 (m, 5H), 3.48 (dd, $1 \mathrm{H}, J=6.4$, $10.5 \mathrm{~Hz}), 1.65-1.58(\mathrm{~m}, 8 \mathrm{H}), 1.43(\mathrm{~m}, 2 \mathrm{H}) .{ }^{13} \mathrm{C}$ NMR $(101 \mathrm{MHz}$, $\left.\mathrm{CDCl}_{3}\right) \delta 138.41,138.27,137.86,137.63,128.43,128.39,128.35$, $128.31,128.29,127.97,127.93,127.85,127.76,127.64,127.59$, 109.85, 106.34 (anomeric carbon), 88.39, 82.74, 80.97, 77.40, $77.09,76.77,76.24,73.99,73.46,73.34,72.10,71.92,70.86$, $67.87,66.49,36.44,35.01,25.19,24.05,23.87$. ESI HRMS Calcd for $\mathrm{C}_{43} \mathrm{H}_{50} \mathrm{O}_{8}$ : $[\mathrm{M}+\mathrm{Na}]^{+} \mathrm{m} / \mathrm{z}$ 717.3398. Found: $[\mathrm{M}+\mathrm{Na}]^{+} \mathrm{m} / \mathrm{z}$ 717.3415.

\subsubsection{3-0-(2,3,5,6-Tetra-0-benzyl- $\beta$-D-galactofuranosyl)-sn- glycerol (14)}

Galactofuranoside $13(2.4 \mathrm{~g}, 3.4 \mathrm{mmol})$ was dissolved in DCM$\mathrm{CH}_{3} \mathrm{CN}(60 \mathrm{~mL}, 1: 1)$ and reacted with ethanediol $(2.4 \mathrm{~mL})$ under acidic condition ( $\mathrm{pH} 1,800 \mathrm{mg}$ of $p$-TsOH); after $5 \mathrm{~h}$ at room temperature, the reaction was quenched by adding sat. $\mathrm{NaHCO}_{3}$ and worked up as usual. Flash silica gel column chromatography (petroleum ether/ethyl acetate $=2: 1)$ gave $14(1.5 \mathrm{~g}, 76 \%)$ as oil. ${ }^{1} \mathrm{H} \mathrm{NMR}\left(400 \mathrm{MHz}, \mathrm{CDCl}_{3}\right): \delta 7.37-7.32(\mathrm{~m}, 20 \mathrm{H}), 5.10(\mathrm{~s}, 1 \mathrm{H}$, anomeric proton), $4.75-4.34(\mathrm{~m}, 8 \mathrm{H}), 4.22(\mathrm{dd}, 1 \mathrm{H}, J=3.8,6.2 \mathrm{~Hz}), 4.05$ $(\mathrm{dd}, 1 \mathrm{H}, J=2.6,6.2 \mathrm{~Hz}), 4.01(\mathrm{~m}, 1 \mathrm{H}), 3.85-3.78(\mathrm{~m}, 2 \mathrm{H}), 3.75-3.57$ $(\mathrm{m}, 6 \mathrm{H}), 2.56$ (br s, 3H). ${ }^{13} \mathrm{C}$ NMR (101 $\left.\mathrm{MHz}, \mathrm{CDCl}_{3}\right) \delta 138.29$,
$138.18,137.58,137.50,128.49,128.44,128.41,128.33,128.30$, $128.06,127.94,127.93,127.89,127.72,127.66,106.20$ (anomeric carbon), 87.65, 82.40, 81.99, 77.41, 77.29, 77.09, 76.77, 76.44, 73.47, 73.31, 72.17, 71.91, 70.49, 70.43, 70.03, 63.85. ESI HRMS Calcd for $\mathrm{C}_{37} \mathrm{H}_{42} \mathrm{O}_{8}$ : $[\mathrm{M}+\mathrm{Na}]^{+} \mathrm{m} / \mathrm{z}$ 637.7138. Found: $[\mathrm{M}+\mathrm{Na}]^{+} \mathrm{m} / \mathrm{z}$ 637.7444 .

\subsubsection{3-O-(2, 3, 5, 6-Tetra-O-benzyl- $\beta$-D-galactofuranosyl)-1,2- di- $O$-myristoyl-sn-glycerol (15)}

Compound $14(1.5 \mathrm{~g}, 2.4 \mathrm{mmol})$ was dissolved in DCM (30 mL) and DCC (1.5 g, $7 \mathrm{mmol}$ ), DMAP (30 mg, $0.24 \mathrm{mmol}$ ) and myristic acid (1.6 g, $7.2 \mathrm{mmol}$ ) were added; after stirring overnight the reaction was quenched by adding $0.2 \mathrm{~mL} \mathrm{MeOH}$ and $0.2 \mathrm{~mL} \mathrm{HOAc}$, then concentrated under reduced pressure and filtered through Celite (washing with petroleum ether $/$ EtOAc $=8: 1$ ). Flash silica gel column chromatography (petroleum ether/ethyl acetate $=$ 10:1) gave $15(2.4 \mathrm{~g}, 98 \%)$ as oil. ${ }^{1} \mathrm{H}$ NMR $\left(400 \mathrm{MHz}, \mathrm{CDCl}_{3}\right): \delta$ 7.27-7.18 $(\mathrm{m}, 20 \mathrm{H}), 5.16(\mathrm{~m}, 1 \mathrm{H}), 4.99(\mathrm{~s}, 1 \mathrm{H}$, anomeric proton), $4.65-4.34(\mathrm{~m}, 7 \mathrm{H}), 4.24-4.28(\mathrm{~m}, 2 \mathrm{H}), 4.10(\mathrm{dd}, 1 \mathrm{H}, J=6.4$, $12.0 \mathrm{~Hz}), 4.03-4.01(\mathrm{~m}, 1 \mathrm{H}), 3.95-3.89(\mathrm{~m}, 2 \mathrm{H}), 3.70-3.66(\mathrm{~m}$, $2 \mathrm{H}), 3.61-3.58(\mathrm{~m}, 2 \mathrm{H}), 3.49(\mathrm{dd}, 1 \mathrm{H}, J=5.0,11.0 \mathrm{~Hz}), 2.23-2.18$ (m, 4H), 1.55-1.50 (m, 4H), $1.18(\mathrm{~m}, 40 \mathrm{H}), 0.81(\mathrm{~m}, 6 \mathrm{H}) .{ }^{13} \mathrm{C} \mathrm{NMR}$ $\left(101 \mathrm{MHz}, \mathrm{CDCl}_{3}\right) \delta 173.40,173.01,138.39,138.29,137.82$, $137.58,128.43,128.37,128.34,128.28,128.27,127.95,127.86$, $127.74,127.63,127.59,106.02$ (anomeric carbon), 88.20, 82.67, $81.18,77.38,77.06,76.74,76.25,73.45,73.33,72.06,71.87$, $70.85,69.72,65.30,62.62,34.34,34.14,31.95,29.72,29.68$, $29.53,29.39,29.33,29.17,29.14,24.97,24.92,22.72,14.15$. ESI HRMS Calcd for $\mathrm{C}_{65} \mathrm{H}_{94} \mathrm{O}_{10}$ : $[\mathrm{M}+\mathrm{Na}]^{+} \mathrm{m} / \mathrm{z}$ 1057.6739. Found: $[\mathrm{M}+\mathrm{Na}]^{+} \mathrm{m} / z 1057.6745$.

\subsubsection{3-O-( $\beta$-D-Galactofuranosyl)-1,2-di-O-myristoyl-sn-glycerol (16)}

$\mathrm{Pd} / \mathrm{C}(100 \mathrm{mg})$ was added to $15(2 \mathrm{~g}, 1.9 \mathrm{mmol})$, TFA $(0.1 \mathrm{~mL})$ in EtOAc-MeOH $(84 \mathrm{~mL}, 20: 1=\mathrm{V}: \mathrm{V})$, and the reaction mixture was stirred overnight under hydrogen atmosphere. The reaction was filtrated through Celite and washed with $\mathrm{MeOH}$. After removal of the solvent under reduced pressure, 16 was obtained as solid (1.1 g, 90\%). $[\alpha]_{D}-19.3$ (c 0.9, $\mathrm{CHCl}_{3}$ ); ${ }^{1} \mathrm{H}$ NMR $\left(400 \mathrm{MHz}, \mathrm{CDCl}_{3}\right)$ : $\delta$ 5.24-5.22 (m, 1H), $5.0(\mathrm{~s}, 1 \mathrm{H}$, anomeric proton), $4.37(\mathrm{dd}, 1 \mathrm{H}$, $J=3.7,12.0 \mathrm{~Hz}), 4.16-4.05(\mathrm{~m}, 4 \mathrm{H}), 3.94(\mathrm{~m}, 1 \mathrm{H}), 3.82-3.75(\mathrm{~m}$, $3 \mathrm{H}), 3.65(\mathrm{dd}, 1 \mathrm{H}, J=5.3,11.0 \mathrm{~Hz}), 3.41(\mathrm{br} \mathrm{s}, 4 \mathrm{H}), 2.35-2.30(\mathrm{~m}$, $4 \mathrm{H}), 1.62-1.60(\mathrm{~m}, 4 \mathrm{H}), 1.27(\mathrm{~m}, 40 \mathrm{H}), 0.89(\mathrm{~m}, 6 \mathrm{H}) .{ }^{13} \mathrm{C} \mathrm{NMR}$ $\left(101 \mathrm{MHz}, \mathrm{CDCl}_{3}\right) \delta 173.70,173.40,108.57$ (anomeric carbon), $86.82,79.68,78.39,77.34,77.03,76.71,70.99,69.80,66.07$, $64.05,62.52,34.30,34.12,31.93,29.71,29.69,29.67,29.52$, $29.37,29.31,29.16,29.12,24.92,24.88,22.70,14.12$. ESI HRMS Calcd for $\mathrm{C}_{37} \mathrm{H}_{70} \mathrm{O}_{10}$ : $[\mathrm{M}+\mathrm{Na}]^{+} \mathrm{m} / \mathrm{z}$ 697.4861. Found: $[\mathrm{M}+\mathrm{Na}]^{+} \mathrm{m} / \mathrm{z}$ 697.4900 .

\subsubsection{3-0-(6-0-tert-Butyldimethylsilyl- $\beta$-D-galactofuranosyl)- 1,2-di-0-myristoyl-sn-glycerol (17)}

Triethylamine ( $0.2 \mathrm{~mL}, 1.2 \mathrm{mmol})$, 4-dimethylaminopyridine (95 mg, $0.8 \mathrm{mmol}$ ) and tert-butyldimethylsilyl chloride (142 $\mathrm{mg}$, $0.9 \mathrm{mmol}$ ) were added to a solution of $16(530 \mathrm{mg}, 0.78 \mathrm{mmol})$ in dry dichloromethane $(4 \mathrm{~mL})$. After stirring for $6 \mathrm{~h}$ at room temperature, the solution was diluted with EtOAC and washed with sat. aqueous $\mathrm{NaHCO}_{3}$ and water and then dried over $\mathrm{MgSO}_{4}$. After concentration in vacuo, the residue was separated by flash silica gel column chromatography (petroleum ether/ethyl acetate = $4: 1-1: 1)$ to give 17 (390 mg, 63\%) as a waxy material. $[\alpha]_{D}-17.3$ (c 1.3, $\left.\mathrm{CHCl}_{3}\right) ;{ }^{1} \mathrm{H}$ NMR $\left(400 \mathrm{MHz}, \mathrm{CDCl}_{3}\right): \delta 5.09(\mathrm{~m}, 1 \mathrm{H}), 4.90$ ( $\mathrm{s}, 1 \mathrm{H}$, anomeric proton), 4.25 (dd, $1 \mathrm{H}, J=4.3,11.8 \mathrm{~Hz}$ ), $4.01-3.99$ $(\mathrm{m}, 2 \mathrm{H}), 3.94(\mathrm{~m}, 1 \mathrm{H}), 3.89(\mathrm{~s}, 1 \mathrm{H}), 3.82(\mathrm{~m}, 1 \mathrm{H}), 3.72(\mathrm{dd}, 1 \mathrm{H}$, $J=5.9,11.0 \mathrm{~Hz}), 3.61(\mathrm{~d}, 1 \mathrm{H}, J=2.3 \mathrm{~Hz}), 3.60(\mathrm{~s}, 1 \mathrm{H}), 3.50(\mathrm{dd}, 1 \mathrm{H}$, $J=4.6,11.0 \mathrm{~Hz}), 2.22-2.19(\mathrm{~m}, 4 \mathrm{H}), 1.52-1.49(\mathrm{~m}, 4 \mathrm{H}), 1.18-1.15$ 
(m, 40H), 0.81-0.76 (m, 15H), $-0.02(\mathrm{~s}, 6 \mathrm{H}) .{ }^{13} \mathrm{C}$ NMR (100 MHz $\mathrm{CDCl}_{3}$ ): $\delta 173.40,173.13,108.75$ (anomeric carbon), 86.72, 78.86, $78.64,77.52,77.48,77.36,77.25,77.21,77.05,76.73,71.74$, $69.77,65.86,63.93,62.25,34.29,34.12,31.96,29.72,29.69$, 29.67. 29.52, 29.39, 29.32, 29.17, 29.13, 25.86, 24.91, 22.72 18.27, 14.15, -5.33. HRMS Calcd for $\mathrm{C}_{43} \mathrm{H}_{84} \mathrm{O}_{10} \mathrm{Si}:[\mathrm{M}+\mathrm{Na}]^{+} \mathrm{m} / \mathrm{z}$ 811.5834. Found: $[\mathrm{M}+\mathrm{Na}]^{+} \mathrm{m} / \mathrm{z} 811.6856$.

\subsubsection{3-0-(6-0-tert-Butyldimethylsilyl-2,3,5-tri-0-benzyl- $\beta$-D- glactofuranosyl)-1,2-di-0-myristoyl-sn-glycerol (18)}

To a solution of $17(560 \mathrm{mg}, 0.7 \mathrm{mmol})$ and benzyl bromide $(0.5 \mathrm{~mL}, 4 \mathrm{mmol})$ in DMF (3 mL) was added $60 \% \mathrm{NaH}(120 \mathrm{mg}$ $3 \mathrm{mmol}$ ). The resulting suspension was stirred for $2 \mathrm{~h}$ at room temperature, poured into ice water $(10 \mathrm{~mL})$, and extracted with EtOAc. The obtained extract was washed with water and brine, dried over $\mathrm{MgSO}_{4}$, and evaporated in vacuo. The residue was purified by silica gel column chromatography (petroleum ether/ethyl acetate $=$ $15: 1)$ to give 18 (700 $\mathrm{mg}, 90 \%)$ as a colourless syrup. $[\alpha]_{\mathrm{D}}-11.6$ (c $\left.1.3, \mathrm{CHCl}_{3}\right) ;{ }^{1} \mathrm{H} \mathrm{NMR}\left(400 \mathrm{MHz}, \mathrm{CDCl}_{3}\right): \delta 7.30-7.21(\mathrm{~m}, 15 \mathrm{H})$ $5.20(\mathrm{~m}, 1 \mathrm{H}), 5.02(\mathrm{~s}, 1 \mathrm{H}$, anomeric proton), $4.65-4.37(\mathrm{~m}, 6 \mathrm{H})$, $4.24-4.20(\mathrm{~m}, 2 \mathrm{H}), 4.16-4.12(\mathrm{~m}, 1 \mathrm{H}), 4.05(\mathrm{dd}, 1 \mathrm{H}, J=2.6$, $7.2 \mathrm{~Hz}), 3.98-3.93(\mathrm{~m}, 2 \mathrm{H}), 3.75-3.71(\mathrm{~m}, 3 \mathrm{H}), 3.54-3.50(\mathrm{~m}, 2 \mathrm{H})$, 2.24-2.19 (m, $4 \mathrm{H}), 1.57-1.54(\mathrm{~m}, 4 \mathrm{H}), 1.20(\mathrm{~m}, 40 \mathrm{H}), 0.87-0.74$ $(\mathrm{m}, 15 \mathrm{H}),-0.02(\mathrm{~s}, 6 \mathrm{H}) .{ }^{13} \mathrm{C}$ NMR $\left(100 \mathrm{MHz}, \mathrm{CDCl}_{3}\right): \delta 173.40$ $172.98,138.51,137.93,137.68,128.45,128.43,128.37,128.34$ $128.30,127.98,127.96,127.92,127.88,127.85,127.82,127.80$ $127.76,127.69,127.66,127.63,127.60,106.17$ (anomeric carbon), $88.34,82.58,80.50,77.42,77.10,76.78,73.67,72.10,71.89,69.75$, $63.28,62.68,34.38,34.18,31.99,29.75,29.72,29.56,29.42,29.36$ $29.21,29.17,25.98,25.01,24.96,22.75,18.31,14.18,-5.33,-5.39$ HRMS Calcd for $\mathrm{C}_{64} \mathrm{H}_{102} \mathrm{O}_{10} \mathrm{Si}:[\mathrm{M}+\mathrm{Na}]^{+} \mathrm{m} / \mathrm{z}$ 1081.6242. Found: $[\mathrm{M}+\mathrm{Na}]^{+} \mathrm{m} / \mathrm{z} 1081.5713$.

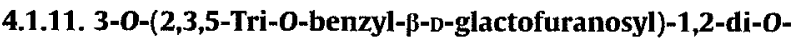 myristoyl-sn-glycerol (19)}

The solution of 18 (900 $\mathrm{mg}, 0.85 \mathrm{mmol})$ in DCM $(5 \mathrm{~mL})$ was treated with TBAF ( $1 \mathrm{M}$ solution in THF, $4 \mathrm{~mL}$ ). The reaction mixture was stirred overnight at room temperature, then diluted with EtOAc and washed with saturated aqueous $\mathrm{NH}_{4} \mathrm{Cl}$ and water, the organic phase was dried over $\mathrm{MgSO}_{4}$ and the solvent was evaporated in vacuo. Purification by silica gel column chromatography (petroleum ether/ethyl acetate $=10: 1)$ yielded $19(600 \mathrm{mg}, 76 \%)$ as a colourless syrup. $[\alpha]_{\mathrm{D}}-15.1$ (c $\left.1.0, \mathrm{CHCl}_{3}\right) ;{ }^{1} \mathrm{H}$ NMR $\left(400 \mathrm{MHz}, \mathrm{CDCl}_{3}\right): \delta 7.31-7.22(\mathrm{~m}, 15 \mathrm{H}), 5.20(\mathrm{~m}, 1 \mathrm{H}), 5.02(\mathrm{~s}$, $1 \mathrm{H}$, anomeric proton), 4.57-4.42 (m, 6H), 4.34-4.27 (m, $2 \mathrm{H})$, 4.16-4.12 (m, 2H), 3.98-3.96 (m, 2H), 3.71-3.56 (m, 5H), 2.28$2.23(\mathrm{~m}, 4 \mathrm{H}), 1.55(\mathrm{~m}, 4 \mathrm{H}), 1.20(\mathrm{~m}, 40 \mathrm{H}), 0.84-0.82(\mathrm{~m}, 6 \mathrm{H}) .{ }^{13} \mathrm{C}$ NMR $\left(101 \mathrm{MHz}, \mathrm{CDCl}_{3}\right) \delta 173.55,173.12,138.22,137.46,137.30$, $128.53,128.45,128.12,128.08,127.93,127.84,106.10$ (anomeric carbon), 87.92, 81.77, 77.37, 77.25, 77.05, 76.73, 72.12, 69.78 $65.41,62.66,61.83,34.16,31.96,29.73,29.69,29.54,29.40$, 29.34, 29.16, 24.98, 24.93, 22.73, 14.16. HRMS Calcd for $\mathrm{C}_{58} \mathrm{H}_{88} \mathrm{O}_{10}:[\mathrm{M}+\mathrm{Na}]^{+} \mathrm{m} / \mathrm{z}$ 967.6270. Found: $[\mathrm{M}+\mathrm{Na}]^{+} \mathrm{m} / \mathrm{z} 967.6251$.

4.1.12. 3-0-[6-0-(Benzyloxy-diisopropylaminophoshinyl)-3,5,6tri-0-benzyl- $\beta$-D-galactofuranosyl]-1,2-di-0-myrisotyl-snglycerol (2)

Bis(diisopropylamino)benzyloxyphosphine ( $165 \mathrm{mg}, 0.5 \mathrm{mmol}$ ) and diispropylammonium tetrazolide $(83 \mathrm{mg}, 0.5 \mathrm{mmol})$ were added to the solution of $19(230 \mathrm{mg}, 0.24 \mathrm{mmol})$ in DCM $(5 \mathrm{~mL})$; the reaction was kept at room temperature under $\mathrm{Ar}$ for $1 \mathrm{~h}$ and then diluted with DCM, washed with saturated aqueous $\mathrm{NaHCO}_{3}$ and water. The organic phase was dried over $\mathrm{MgSO}_{4}$ and the solvent was evaporated in vacuo below $30^{\circ} \mathrm{C}$. Purification by flash silica gel column chromatography (petroleum ether $/ \mathrm{NEt}_{3}=8: 1$ ) yielded 2 as oil ( $230 \mathrm{mg}, 81 \%$ ). This mixture of diastereomers was immediately used in the reaction with 3 to afford 20 .

\subsubsection{Compound 20}

To a solution of glycerol moiety $3(48 \mathrm{mg}, 0.03 \mathrm{mmol}$ ) and phosphite $2(50 \mathrm{mg}, 0.04 \mathrm{mmol})$ in DCM $(2 \mathrm{~mL})$, tetrazole $(0.2 \mathrm{~mL}$, $0.45 \mathrm{M}$ in $\mathrm{CH}_{3} \mathrm{CN}$ ) was added; the reaction mixture was stirred for $6 \mathrm{~h}$ under $\mathrm{Ar}$ and then ${ }^{t} \mathrm{BuOOH}(20 \mu \mathrm{L}, 5.4 \mathrm{M}$ in decane) was added. After $2 \mathrm{~h}$, the mixture was diluted with DCM and washed with saturated aqueous $\mathrm{NaHCO}_{3}$ and water. The organic phase was dried over $\mathrm{MgSO}_{4}$ and the solvent was evaporated in vacuo at less than $30^{\circ} \mathrm{C}$. Purification by flash silica gel column chromatography (toluene/acetone $=3: 1$ ) yielded the phosphate $\mathbf{2 0}$ as oil (70 mg, $90 \%$, mixture of diastereomers). ${ }^{1} \mathrm{H} \mathrm{NMR}\left(400 \mathrm{MHz}, \mathrm{CDCl}_{3}\right)$ : $\delta 7.37-7.21(\mathrm{~m}, 70 \mathrm{H}), 6.82(\mathrm{~m}, 8 \mathrm{H}), 5.27(\mathrm{~m}, 1 \mathrm{H}), 5.04-5.01(\mathrm{~m}$, $10 \mathrm{H}$, include anomeric proton), 4.71-4.48 $(\mathrm{m}, 17 \mathrm{H}), 4.33-3.99$ $(\mathrm{m}, 24 \mathrm{H}), 3.76-3.56\left(\mathrm{~m}, 21 \mathrm{H}\right.$, include $\left.\mathrm{CH}_{3}-\mathrm{O}-\mathrm{Ph}\right), 2.34-2.31(\mathrm{~m}$, $4 \mathrm{H}), 1.63(\mathrm{~m}, 4 \mathrm{H}), 1.30(\mathrm{~m}, 40 \mathrm{H}), 0.94-0.92(\mathrm{~m}, 6 \mathrm{H}) .{ }^{13} \mathrm{C}$ NMR $\left(101 \mathrm{MHz}, \mathrm{CDCl}_{3}\right) \delta 173.38,172.98,159.36,138.16,138.03$, $137.91,137.64,137.45,135.72,129.65,129.54,128.62,128.48$, $128.42,128.39,128.35,128.21,127.98,127.94,127.79,127.70$ 127.64, 113.83, 106.17 (anomeric carbon), 87.77, 77.40, 77.29, $77.08,76.77,75.45,73.45,72.24,72.08,71.93,69.65,69.47$, $69.13,65.89,62.61,55.22,34.34,34.14,31.96,29.73,29.69$, $29.55,29.40,29.35,29.19,29.16,24.98,24.93,22.73,14.16$. ESI HRMS (neg. ion mode) Calcd for $\mathrm{C}_{154} \mathrm{H}_{197} \mathrm{O}_{39} \mathrm{P}_{5}:[\mathrm{M}-\mathrm{Bn}]^{-} \mathrm{m} / \mathrm{z}$ 2734.1578. Found: $m / z 2734.1578$.

\subsubsection{Compound 21}

At $-10^{\circ} \mathrm{C}, \mathrm{Ce}\left(\mathrm{NH}_{4}\right)_{2}\left(\mathrm{NO}_{3}\right)_{6}(124 \mathrm{mg}, 0.23 \mathrm{mmol})$ was added portion wise to a solution of 20 ( $80 \mathrm{mg}, 0.028 \mathrm{mmol}$ ) in $\mathrm{CH}_{3} \mathrm{CN} /$ toluene $/ \mathrm{H}_{2} \mathrm{O}(60: 3: 4,10 \mathrm{~mL})$, the reaction was stirred for $30 \mathrm{~min}$ (TLC-monitoring) and then diluted with EtOAC and washed with saturated $\mathrm{NaHCO}_{3}$ solution. The organic phase was dried over $\mathrm{MgSO}_{4}$ and the solvent was evaporated in vacuo at less than $30^{\circ} \mathrm{C}$. Purification by flash silica gel column chromatography (toluene/acetone $=1: 1$ ) yielded 21 as oil $(60 \mathrm{mg}, 85 \%$, mixture of diastereomers). ${ }^{1} \mathrm{H}$ NMR $\left(400 \mathrm{MHz}, \mathrm{CDCl}_{3}\right): \delta 7.37-7.21(\mathrm{~m}, 50 \mathrm{H})$ $5.26(\mathrm{~m}, 1 \mathrm{H}), 5.08(\mathrm{~m}, 12 \mathrm{H}), 4.64-4.61(\mathrm{~m}, 12 \mathrm{H}), 4.33-3.99(\mathrm{~m}$, $30 \mathrm{H}), 3.78-3.58(\mathrm{~m}, 12 \mathrm{H}), 2.34-2.30(\mathrm{~m}, 4 \mathrm{H}), 1.62(\mathrm{~m}, 4 \mathrm{H}), 1.30$ $(\mathrm{m}, 40 \mathrm{H}), 0.94-0.92(\mathrm{~m}, 6 \mathrm{H})$. ESI HRMS (neg. ion mode) Calcd for $\mathrm{C}_{122} \mathrm{H}_{165} \mathrm{O}_{35} \mathrm{P}_{5}:[\mathrm{M}-\mathrm{Bn}]^{-} \mathrm{m} / z$ 2253.9177. Found: $m / z 2253.9277$.

\subsubsection{Compound 22}

$\mathrm{N}$-Cbz-D-Alanine triethylammonium salt $(82 \mathrm{mg}, 0.26 \mathrm{mmol}$ ) and PyBOP (131 mg, $0.26 \mathrm{mmol}$ ) were added to a solution of 21 (40 mg, $0.013 \mathrm{mmol})$ in dry DCM $(4 \mathrm{~mL})$ under Ar, and then $N$ methylimidazole $(40 \mu \mathrm{L}, 0.52 \mathrm{mmol})$ was added drop wise. After $5 \mathrm{~h}$, the reaction mixture was diluted with DCM and washed with saturated $\mathrm{NH}_{4} \mathrm{Cl}$ solution. The organic phase was dried over $\mathrm{MgSO}_{4}$ and the solvent was evaporated in vacuo at less than $30^{\circ} \mathrm{C}$. Purification by flash silica gel column chromatography (toluene/acetone $=3: 1-1: 1$ ) yielded a diastereomeric mixture of 22 as oil ( $40 \mathrm{mg}, 74 \%$, mixture of diastereomers). ${ }^{7} \mathrm{H}$ NMR ( $\left.400 \mathrm{MHz}, \mathrm{CDCl}_{3}\right)$ : $\delta 7.34-7.21(\mathrm{~m}, 70 \mathrm{H}), 5.26(\mathrm{~m}, 1 \mathrm{H}), 5.07-5.03(\mathrm{~m}, 20 \mathrm{H}), 4.63-4.61$ $(\mathrm{m}, 4 \mathrm{H}), 4.58-3.98(\mathrm{~m}, 35 \mathrm{H}), 3.75-3.56(\mathrm{~m}, 10 \mathrm{H}), 2.32-2.30(\mathrm{~m}$, $4 \mathrm{H}), 1.62(\mathrm{~m}, 4 \mathrm{H}), 1.36-1.28(\mathrm{~m}, 52 \mathrm{H}), 0.94-0.90(\mathrm{~m}, 6 \mathrm{H})$. ESI HRMS (neg. ion mode) Calcd for $\mathrm{C}_{166} \mathrm{H}_{209} \mathrm{~N}_{4} \mathrm{O}_{47} \mathrm{P}_{5}$ : [M-Bn] ${ }^{-} \mathrm{m} / \mathrm{z}$ 3074.2233. Found: $m / z 3074.1780$.

\subsubsection{Compound 1a}

The diasteromers of $\mathbf{2 2}(40 \mathrm{mg}, 0.013 \mathrm{mmol})$ were dissolved in $\mathrm{DCM} / \mathrm{MeOH} / \mathrm{H}_{2} \mathrm{O}(7: 3: 2,24 \mathrm{~mL})$ and then treated with Pearlman's catalyst $(5 \mathrm{mg}$ ) under a hydrogen atmosphere. After stirring overnight at room temperature, the reaction mixture was filtrated through Celite, washed with $\mathrm{DCM} / \mathrm{MeOH} / \mathrm{H}_{2} \mathrm{O}(7: 3: 2)$, and the 
filtrate was concentrated under reduced pressure below $30^{\circ} \mathrm{C}$. The crude residue (1a) was purified with hydrophobic interaction chromatography (HIC) based on the protocol of W. Fischer ${ }^{19}$ with the following modifications. Briefly, the compound was dispersed in $0.5 \mathrm{~mL} 50 \mathrm{mM} \mathrm{NH}_{4} \mathrm{OAc} \mathrm{pH}$ 4.7/n-propanol 85:15 (v/v) (solvent A for HIC) under ultrasonication ( $5 \mathrm{~min}$ ). The procedure was repeated twice and the resulting $1.5 \mathrm{~mL}$ suspension was centrifuged (Biofuge Heraeus, $13,000 \mathrm{rpm}, 5 \mathrm{~min}$, room temperature). The sediment was dissolved three times in $0.5 \mathrm{~mL}$ solvent $A$ and again centrifuged. The resulting clear $2.5 \mathrm{~mL}$ supernatant was divided into two aliquots (1.25 mL each) and injected for two hydrophobic interaction chromatography (HIC) runs. The HIC system (Gilson) was equipped with a HiPrep $16 / 10$ octyl agarose column $(16 \times 100 \mathrm{~mm}$. Amersham Pharmacia Biotech). The HIC operated at a flow of $0.5 \mathrm{~mL} / \mathrm{min}$ (47 bar) solvent $A$ isocratic $(0 \%$ B) for $75 \mathrm{~min}$, then raised in 250 min linear to $100 \%$ solvent $\mathrm{B}\left[50 \mathrm{mM} \mathrm{NH}_{4} \mathrm{OAc} \mathrm{pH} 4.7 / n\right.$-propanol 40:60 (v/v)] staying isocratic for additional $75 \mathrm{~min}$ at $100 \% \mathrm{~B}$. The synthetic LTA was monitored by its UV absorbance $(254 \mathrm{~nm})$ and fractions $(3 \mathrm{~mL})$ were collected. Aliquots of $30 \mu \mathrm{L}$ were tested for organic phosphate by a photometric test. ${ }^{20}$ The phosphate positive fractions were combined that gave $6.3 \mathrm{mg}(28 \%)$ of pure 1a. For the NMR data see Table 1.-ESI HRMS (neg. ion mode) Calcd for $\mathrm{C}_{64} \mathrm{H}_{124} \mathrm{~N}_{4} \mathrm{O}_{39} \mathrm{P}_{5}[\mathrm{M}-\mathrm{H}]^{-} m / z$ 1727.5607. Found: $m / z$ 1727.6639. Besides this compound in the ESI-FT-ICR mass spectrum (see Supplementary data) further species of LTA with different degree in alanylation are present: completely de-alanylated $\mathrm{m} / \mathrm{z}$ 1443.4973 (rel. intensity 10\%), (ii), mono-alanylated $\mathrm{m} / \mathrm{z}$ 1514.5413 (13\%), di-alanylated $m / z 1585.5783$ (40\%), tri-alanylated $\mathrm{m} / \mathrm{z} 1656.6066$ (30\%), These data reflect the degree in de-alanylation since they are also in agreement with the NMR analysis, in which also this kind and degree of heterogeneity in the D-alanylation was observed.

\section{Acknowledgements}

This work was supported by the Fonds der Chemischen Industrie and by LUNAMed AG, Switzerland. We wish to thank B. Wegner,
H. Moll, H. Käßner and Dr. N. Gisch for excellent LTA purification and help in MS and NMR analysis.

\section{References and notes}

1. Roethlisberger, P.; lida-Tanaka, N.; Hollemeyer, K.; Heinzle, E.; Ishizuka, I.; Fischer, W. Eur, J. Biochem. 2000, 267, 5520.

2. Roethlisberger, P. Ph. D Thesis, ETH Zurich, Switzerland, 1995.

3. (a) Fischer, W.; Behr, T; Hartmann, R.; Peter-Katalinié, J.; Egge, H. Eur. J. Biochem. 1993, 215, 851; (b) Greenberg, J. W.; Fischer, W.; Joiner, K. A. Infect. Immun, 1996, 64, 3318; (c) Fischer, W. Microb. Drug. Resist. 1997, 3, 309.

4. Pedersen, C. M.; Figueroa-Perez, I.; Lindner, B.; Ulmer, A. J.; Zähringer, U. Schmidt, R. R. Angew. Chem., Int. Ed. 2010, 49, 2583.

5. Umland, O.; Heine, H.; Miehe, M.; Marienfeld, K.; Staubach, K. H.; Ulmer, A. J.J. Leukocyte Biol. 2004, 75, 671.

6. Morath, S.; Geyer, A.; Hartung, T. J. Exp. Med. 2001, 193, 393.

7. Stadelmaier, A.; Morath, S.; Hartung, T.; Schmidt, R. R. Angew. Chem 2003, 115 , 945. Angew. Chem., Int. Ed. 2003, 42, 916.

8. This compound is commercially available.

9. Figueroa-Perez, I.; Stadelmaier, A.; Morath, S.; Hartung, T.; Schmidt, R. R. Tetrahedron: Asymmetry 2005, 16, 493.

10. Baeschlin, D. K.; Chaperon, A. R.; Charbonneau, V.; Green, L. G.; Ley, S. V.; Lücking, U.: Walther, E. Angew. Chem. 1998, 110, 3609. Angew. Chem., Int. Ed. 1998, 37, 3423 .

11. Wickberg, B. Acta Chem. Scand. 1958, 12, 1187

12. Ferrières, V.; Bertho, J.-N.; Plusquellec, D. Carbohydr. Res. 1998, 311, 25.

13. Ferrières, V.; Roussel, M.; Gelin, M.; Plusquellec, D.J. Carbohydr. Chem. 2001, 20. 855.

14. Tsvetkov, Y. E.; Nikolaev, A. V. J. Chem. Soc., Perkin Trans. 1 2000, 889.

15. (a) Choudhury, A. K.; Roy, N. Carbohydr. Res. 1998, 308, 207; (b) Marlow, A. K.; Kiessling, L. L. Org. Lett. 2001, 3, 2517.

16. Wang, H.; Ning, J. Carbohydr. Res. 2003, 338, 1033.

17. (a) Gandolfi-Donadílo, L.; Gola, G.; de Lederkremer, R. M.; Gallo-Rodriguez, C. Carbohydr. Res. 2006, 341, 2487; (b) Gallo-Rodriguez, C.; Gil-Libarona, M. A.; Meridoza, V. M.; de Lederkremer, R. M. Tetrahedron 2002, 38, 9373; (c) Completo, G. C.; Lowary, T. L. J. Org. Chem. 2008, 73, 4513; (d) Lee, Y. J.; Lee, B.Y.; Jeon, H. B.: Kim, K. S. Org. Lett. 2006, 8, 3971.

18. (a) Bannwarth, W.; Trzeciak, A. Helv. Chim. Acta 1987, 70, 175; (b) Kratzer, B. Schmidt, R. R. Liebigs Ann, 1995, 957.

19. Fischer, W. Anal. Biochem. 1991, 194, 353.

20. Strominger, J. L.; Park, J. T.; Thompson, R. E. I. Biol Chem. 1959, 234, 3263. 